\title{
Grain Refinement and Delta Ferrite Reduction of High Cr Steel Ingots by Thermal Control
}

\author{
Long ZENG, Mingqin XU, Xiaoran MA, Yujin HUANG, Shuguang ZHANG, Qiaodan HU and Jianguo LI* \\ School of Materials Science and Engineering, Shanghai Jiao Tong University, Shanghai, 200240 P.R. China. \\ (Received on March 7, 2014; accepted on June 3, 2014)
}

\begin{abstract}
High Cr steel (X12CrMoWVNbN10-1-1) ingots were prepared by thermal control by varying three parameters: the melt pouring temperature, the mold temperature and the melt superheating temperature. The effects of these parameters on the macro-/micro-structure of the ingots were investigated through a group of orthogonal experiments. The results showed that superheating temperature was the dominating parameter that affects the grain size and fraction of delta ferrite. The optimized parameters for the sound ingot were $1893 \mathrm{~K}$ for superheating temperature, $1073 \mathrm{~K}$ for mold temperature and $1873 \mathrm{~K}$ for pouring temperature. Under that condition, the ingot was composed of fine equiaxed grain with a radius of 1.1 $\mathrm{mm}$, and the fraction of the delta ferrite was less than $2 \%$. Mechanisms that explain the increment in the proportion of the equiaxed fraction and the grain refinement were proposed, and the mechanisms for the delta ferrite reduction were explained.
\end{abstract}

KEY WORDS: martensitic steel; casting; grain refinement; delta ferrite.

\section{Introduction}

The size of rotor shaft forgings has increased significantly in recent years and is certain to increase further in the future because large forgings allow the integrated advanced design of the steam turbines and reactor pressure vessel components, which increases the safety and reliability of the power stations. According to Tanaka and Sato, ${ }^{1)}$ the properties that are required of large forgings are homogeneity, high fracture toughness, resistance to aging embrittlement and environmental damage, among others. To produce sound large forgings, a homogeneous ultra-large ingot with fine grains is needed.

Usually, three basic ways are used to obtain grain refinement during the solidification process: chemical stimulation, physical means or thermal controls. In the chemical refinement approach, inoculants with a lower melting point than the matrix alloy are added to the alloy. Nakajima et $a .^{2)}$ examined the catalytic potency of the three inoculants (including $\mathrm{Al}_{2} \mathrm{O}_{3}, \mathrm{Ti}_{2} \mathrm{O}_{3}$ and $\mathrm{TiN}$ ) in pure $\mathrm{Fe}$ and $\mathrm{Fe}-\mathrm{Ni}$ alloys using differential scanning calorimetry (DSC) and proved that the TiN's was the greatest. Poole et al. ${ }^{3)}$ investigated the effectiveness of TiN particles in ferritic stainless steel and found that it was very effective in promoting heterogeneous nucleation. However, the particle size was approximately $10 \mu \mathrm{m}$, and it had a harmful effect on the steel properties because these coarse inclusions could act as nucleation sites for fatigue crack initiation. For the alloys used for rotor shafts, they cannot be chemically grain refined because any deviation from their chemical compo-

* Corresponding author: E-mail: lijg@sjtu.edu.cn

DOI: http://dx.doi.org/10.2355/isijinternational.54.2302 sitions could lead to unpredictable changes in their properties. In the physical refinement approach, an external force is introduced to melt during solidification, which breaks up the dendrites and produces more nucleation sites. The electromagnetic process and ultrasonic treatment have been proven to be simple physical methods. Zhang et al. ${ }^{4)}$ studied numerically the effects of the electromagnetic intensity on the electromagnetic field, fluid flow, temperature field and solidification during a low-frequency electromagnetic casting (LFEC) process. These authors verified that the grain refinement induced by the electromagnetic field was limited by the magnetic intensity and that fine microstructures in 7XXX series aluminum alloys could be gained when the electromagnetic intensity was more than 6400 AT (ampere turn, which is used to denote the electromagnetic intensity). Ma et $a l .{ }^{5)}$ found that the K417 alloy was significantly refined by pulsed magnetics, and the grains in the center were much coarser than those near the surface because of a skin effect. Liu et al. ${ }^{6)}$ studied the effect of power ultrasonic treatment on the microstructure of T10 steel. The refinement effect of the ultrasonic treatment was closely related to the location of the ultrasonic vibration generator, and it was less efficient outside the region of active cavitation. From this research, it was concluded that physical refinement could not obtain a homogeneous structure in a large ingot. With respect to thermal control, it is easy to implement them through varying the casting parameters, such as the mold temperature, melt pouring temperature, melt superheating temperature and cooling rate, and several superalloys have been successfully cast. Michael and Howard ${ }^{7)}$ produced integrally cast turbine wheels using Mar-M247 and IN713LC alloys, which possess fine grain structures. 
In this work, $\mathrm{X} 12 \mathrm{CrMoWVNbN10-1-1} \mathrm{(refer} \mathrm{to} \mathrm{X12}$ next) steel ingots were prepared by thermal control, and the effects of casting parameters on the macro-structure were investigated. X12 steel has been widely used for rotor shafts because of its high temperature strength, high temperature corrosion resistance and low thermal expansion coefficient. However, martensitic steels have a high content of chromium incline to form delta ferrite frequently, which influences the properties of these steels. Kumar et al. ${ }^{8)}$ demonstrated that the presence of delta ferrite deteriorated the overall hardness of $9 \mathrm{Cr}-1 \mathrm{Mo}$ steel, and Sawada et al. ${ }^{9)}$ reported that it had a negative influence on the long-term creep strength of TP91 steel. To avoid its presence, the effects of the casting parameters on the fraction of delta ferrite were also investigated with respect to how it was governed by the chemical composition and parameters.

\section{Experimental}

In this study, the chemical composition (wt.\%) of the X12 steel was as follows: $0.12 \mathrm{C}, 10.5 \mathrm{Cr}, 1.06 \mathrm{Mo}, 0.98 \mathrm{~W}, 0.45$ $\mathrm{Mn}, 0.18 \mathrm{~V}, 0.055 \mathrm{Nb}, 0.75 \mathrm{Ni}, 0.052 \mathrm{~N}, 0.09 \mathrm{Si}$ and others. An experimental design technique called orthogonal experiments was used to determine the optimum range of the various parameters. The basic principle of this method is to develop an understanding of the individual and combined effects of various design parameters from a minimum number of experiments. ${ }^{10)}$ The three influential casting parameters were selected, i.e., the pouring temperature $\left(T_{p}\right)$, mold temperature $\left(T_{m}\right)$ and melt superheating temperature $\left(T_{s}\right)$, with three levels for each parameter, as listed in Table 1. Melt

Table 1. Parameters and levels selected for experiments.

\begin{tabular}{cccc}
\hline Level & $T_{p}(\mathrm{~K})$ & $T_{m}(\mathrm{~K})$ & $T_{s}(\mathrm{~K})$ \\
\hline 1 & 1853 & 673 & 1893 \\
2 & 1873 & 873 & 1973 \\
3 & 1893 & 1073 & 2053 \\
\hline
\end{tabular}

superheating temperature is an important factor during melt superheating treatment which involves heating a molten alloy to it, holding the melt for a required period, and then cooling rapidly to the require pouring temperature. It was set in the range of $1893-2053 \mathrm{~K}$ in this experiment which was in the range of 100-300 $\mathrm{K}$ above liquidus of the alloy suggested by Pei et al. ${ }^{11)}$ The parameters and levels were used to set up a standard L9 $\left(3^{4}\right)$ orthogonal matrix and nine ingots were cast, as shown in Table 2.

The casting experiments were performed in a vacuum melting and casting unit, as shown in Fig. 1. The experimental apparatus was mainly an electric resistance furnace and a medium frequency induction melting furnace, both of which were installed in a stainless steel chamber. For the sake of simplicity, the experiment procedure was summarized as follows:

(i) The casting mold was heated to $T_{m}$ by the electric resistance furnace.

(ii) The chamber was evacuated and then filled with argon.

(iii) The alloy was heated to $T_{s}$ by the medium frequency induction melting furnace.

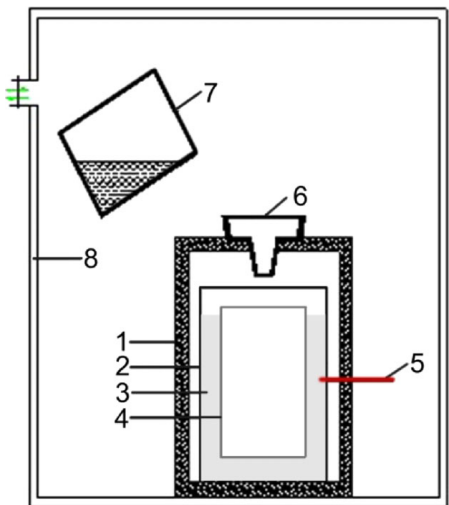

Fig. 1. Schematic of experimental device (1. electric resistance furnace; 2 . stainless steel vessel; 3 . sand; 4 . mold; 5 . thermocouple; 6 . pouring cup; 7 . induction melting furnace; 8 . chamber.) (Online version in color.)

Table 2. Trail conditions and the corresponding results for the orthogonal experiments.

\begin{tabular}{|c|c|c|c|c|c|c|}
\hline \multirow{2}{*}{ No. } & \multicolumn{3}{|c|}{ Parameters } & \multicolumn{3}{|c|}{ Results } \\
\hline & $T_{p}(\mathrm{~K})$ & $T_{m}(\mathrm{~K})$ & $T_{s}(\mathrm{~K})$ & PEGZ (\%) & GS (um) & FDF $(\%)$ \\
\hline 1 & 1853 & 673 & 1893 & 13.02 & 3285 & 3.7 \\
\hline 2 & 1853 & 873 & 1973 & 17.71 & 2725 & 5.7 \\
\hline 3 & 1853 & 1073 & 2053 & 38.61 & 3937 & 7.4 \\
\hline 4 & 1873 & 873 & 1973 & 18.42 & 3157 & 4.6 \\
\hline 5 & 1873 & 673 & 2053 & 9.14 & 3244 & 8.1 \\
\hline 6 & 1873 & 1073 & 1893 & 57.01 & 1120 & 1.9 \\
\hline 7 & 1893 & 873 & 2053 & 19.41 & 3712 & 7.3 \\
\hline 8 & 1893 & 673 & 1893 & 23.11 & 2860 & 2.8 \\
\hline 9 & 1893 & 1073 & 1973 & 20.87 & 4144 & 7.2 \\
\hline$K_{l}$ & $\left(T_{p 1}+T_{p 2}+T_{p 3}\right) / 3$ & $\left(T_{m l}+T_{m 4}+T_{m 7}\right) / 3$ & $\left(T_{s 1}+T_{s 6}+T_{s 8}\right) / 3$ & \multirow{3}{*}{\multicolumn{3}{|c|}{$\begin{array}{l}K_{i} \text { is the mean value of the result } \\
\text { under the responding parameter }\end{array}$}} \\
\hline$K_{2}$ & $\left(T_{p 4}+T_{p 5}+T_{p 6}\right) / 3$ & $\left(T_{m 2}+T_{m 5}+T_{m 8}\right) / 3$ & $\left(T_{s 2}+T_{s 4}+T_{s 9}\right) / 3$ & & & \\
\hline$K_{3}$ & $\left(T_{p 7}+T_{p 8}+T_{p 9}\right) / 3$ & $\left(T_{m 3}+T_{m 6}+T_{m 9}\right) / 3$ & $\left(T_{s 3}+T_{s 5}+T_{s 7}\right) / 3$ & & & \\
\hline$R$ & & & & \multicolumn{3}{|c|}{$R$ is the impact factor } \\
\hline
\end{tabular}


(iv) The molten melt was rapidly cooled down to $T_{p}$ and poured into the casting mold after holding at $T_{s}$ for $3 \mathrm{~min}$.

The casting mold was made of sand and Silica sol and coated with emery powder, with an inner diameter of 100 $\mathrm{mm}$. At each heat, approximately $7.5 \mathrm{~kg} \mathrm{X12}$ steel was melted, and the pressure in the chamber was $0.06 \mathrm{MPa}$.

The macrostructure observation of the X12 steel ingots was conducted on the half longitudinal section etched with aqua regia. The proportion of the equiaxed grain zone (PEGZ) and the equiaxed grain size were measured by the linear intercept method according to the ASTM standard E112-96. The columnar grain size was calculated by the following square root equation:

$$
d=(A / n)^{0.5}
$$

Where $A$ is the observed area, and $n$ is the number of the columnar grain.

For the microstructure observation, a specimen with the size $10 \times 10 \mathrm{~mm}$ was cut in the cross section from the ingot, which was $60 \mathrm{~mm}$ from the bottom and $20 \mathrm{~mm}$ from the edge. The specimen was mechanically and electrochemically polished in a mixed solution of $\mathrm{H}_{2} \mathrm{SO}_{4}(55 \mathrm{vol} \%)$ : $\mathrm{HPO}_{4}$ (25 vol\%): $\mathrm{H}_{2} \mathrm{O}(20 \mathrm{vol} \%$ ) to delineate the delta ferrite. The temperature of the solution was $328 \mathrm{~K}$, the electrolytic voltage was $3.5 \mathrm{~V}$, and the electrolytic reaction time was 30-60 s. To measure the fraction of delta ferrite (FDF), the Viella etchant reagent was also used, and 10 fields at least were randomly chosen along the surface of the sample.

\section{Results}

The half longitudinal section morphology of the X12 steel ingots obtained in this orthogonal experiment is shown in Fig. 2. It is obvious that there are a center pipe and some shrinkage porosity in the center of each ingot. From the center pipe, the entire section can be divided into two different zones. One section is the coarse columnar grain zone at the top of the ingot, which closes to the casting cup and owns a higher temperature gradient than the other directions. When the solidification began, the grains first nucleated at the top and the molten melt could not feed in the center of
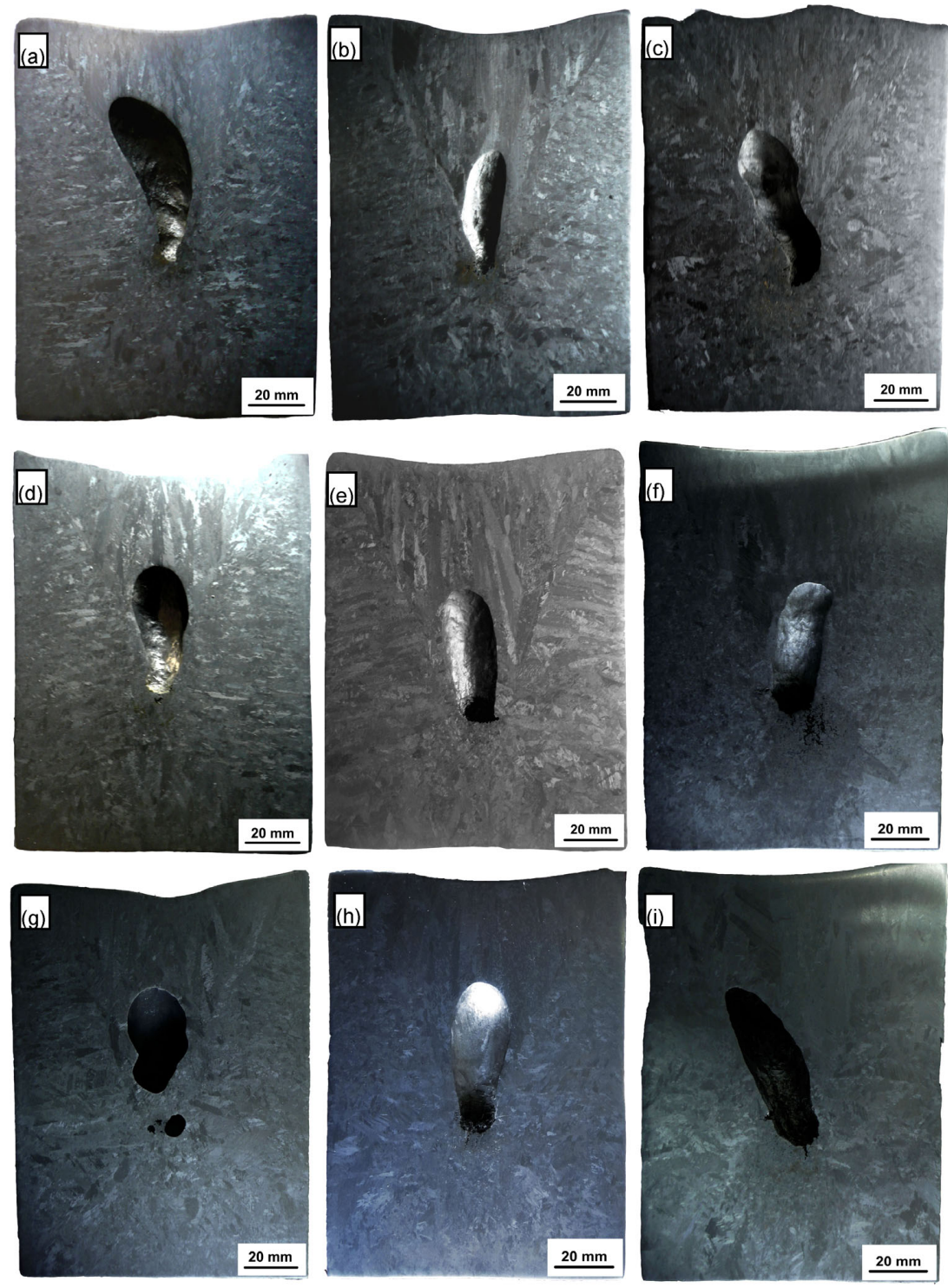

Fig. 2. Longitudinal section morphology of $\mathrm{X} 12$ steel ingots cast in the orthogonal experiment. $T_{p}(\mathrm{~K}) / T_{m}(\mathrm{~K}) / T_{s}(\mathrm{~K})$ : (a) $1853 / 673 / 1893$, (b) $1853 / 873 / 1973$, (c) $1853 / 1073 / 2053$, (d) $1873 / 673 / 1973$, (e) $1873 / 873 / 2$ 053, (f) $1873 /$ 1 073/1 893, (g)1 893,673/2 053, (h)1 893/873/1 893, (i)1 893/1 073/1 973. (Online version in color.) 
Table 3. Effects of parameters on the proportion of equiaxed grain zone and grain size.

\begin{tabular}{cccc}
\hline & $T_{p}(\mathrm{~K})$ & $T_{m}(\mathrm{~K})$ & $T_{s}(\mathrm{~K})$ \\
\hline$K_{l}$ & $23.1 / 3310$ & $16.9 / 3390$ & $31.1 / 2420$ \\
$K_{2}$ & $28.2 / 2500$ & $16.6 / 2940$ & $19.0 / 3340$ \\
$K_{3}$ & $21.1 / 3570$ & $38.8 / 3070$ & $22.5 / 3630$ \\
$R$ & $7.1 / 1070$ & $22.2 / 440$ & $12.1 / 1210$ \\
\hline
\end{tabular}
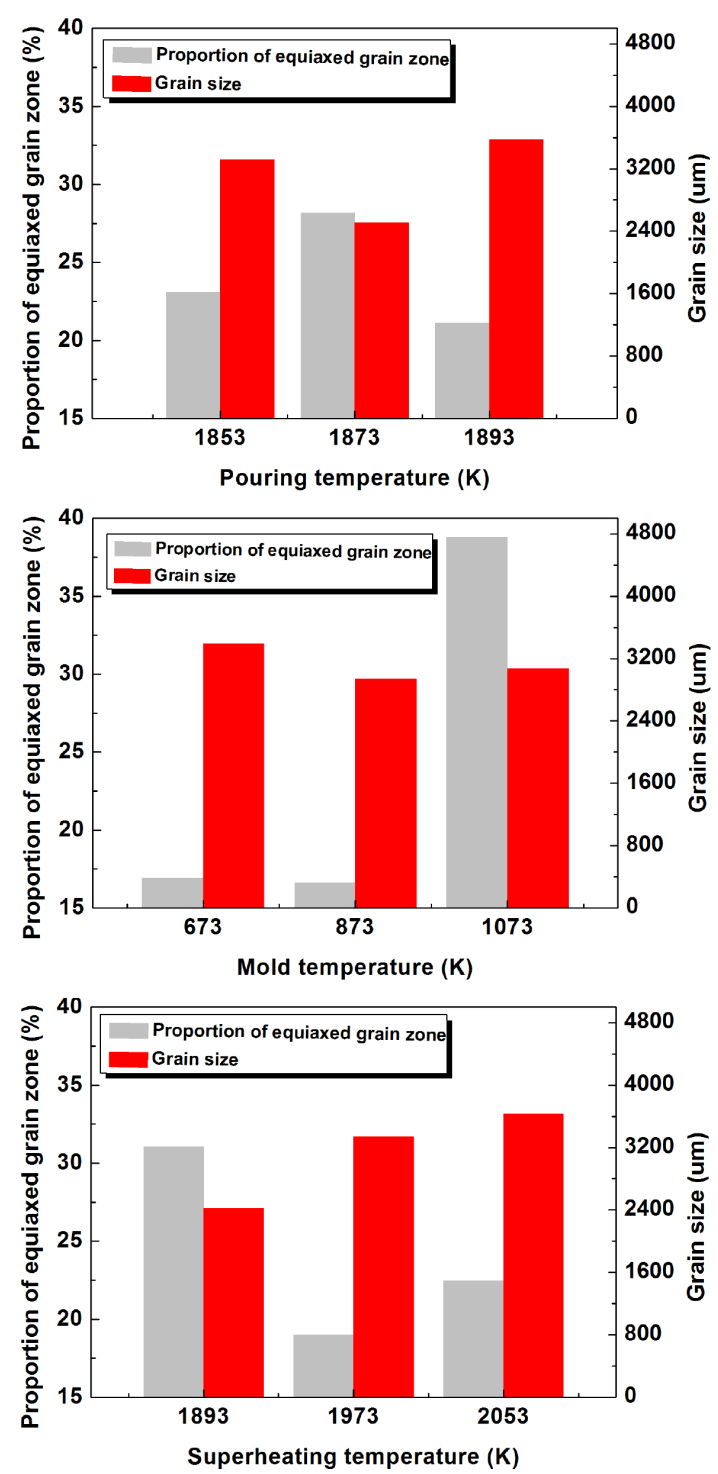

Fig. 3. Effects of parameters on the proportion of equiaxed grain zone and grain size. (Online version in color.)

the ingot; then the center pipe and shrinkage porosity formed. This step can be eliminated by adding a riser or covering agent according to Cui and Liu, ${ }^{12)}$ and no more attention will be paid to it. The second section is the other zone, in which the grains are much smaller than those of the top zone. It should be noted that the proportion of the equiaxed grain zone was identified in the whole section, while the grain size was measured only in the second zone.

Table 2 shows the experimental results, i.e., the proportion of equiaxed grain zone, the grain size and the fraction of delta ferrite. $K_{i}(i=1,2,3)$ means the average value of level $i$, which can be deduced by calculating the results of the orthogonal experiment, and $R$ is the impact factor of the three parameters.

The effects of the three parameters on the proportion of equiaxed grain zone are shown in Table 3. Figure 3 is drawn based on Table 3. From Fig. 3, we can see that the mold temperature is the main factor effect on the proportion of the equiaxed grain zone, and the proportion of the equiaxed grain zone increases with increasing mold temperature. The influences of these three factors in descending order are: mold temperature, melt superheating temperature and pouring temperature, as shown in Table 3. The optimized parameters for larger equiaxed grain zone are: mold temperature of $1073 \mathrm{~K}$, melt pouring temperature of $1873 \mathrm{~K}$ and melt superheating temperature of $1893 \mathrm{~K}$.

The effect of the three parameters on the grain size is also shown in Fig. 3. It can be observed that the melt superheating temperature and the pouring temperature are the main factors that affect the grain size. The mold temperature has little effect on the grain size. The influences of these three factors in descending order are: the melt superheating temperature, the pouring temperature and the mold temperature, as shown in Table 3. The optimized parameters for finer grain are: the melt superheating temperature of $1893 \mathrm{~K}$ the pouring temperature of $1873 \mathrm{~K}$ and the mold temperature of $873 \mathrm{~K}$.

The microstructure of as-cast X12 steel, which is electrochemically polished, is presented in Fig. 4. The etchant did not reveal the martensitic structure but instead revealed the boundaries of the ferrite regions. It is obvious that the microstructure is composed of dendrites of prior austenite, which has transformed to martensite, ${ }^{13)}$ and coarse delta ferrite presented in the interdendritic regions (continuous network). Note that the dendritic structure was broken up, and the continuous network of delta ferrite in the interdendritic region was also interrupted in Fig. 5(f), where the grain is equiaxed and finer. Furthermore, the fraction of delta ferrite varies acutely at different casting parameters, as seen in Fig. 5 . Hence, it is necessary to investigate the effect of the casting parameters on the fraction of delta ferrite. Figure 6 shows the same material etched with Viella etchant reagent, also in the as-cast condition. In these images, the white region is the delta ferrite, and the dark region is the martensite.

The effect of the three parameters on the fraction of delta ferrite is shown in Table 4. Figure 6 is drawn based on Table 4. The fraction of delta ferrite decreases when the melt superheating temperature is decreased, but little affect is noted in the mold temperature. The fraction of delta ferrite decreases when the pouring temperature increases to 1873 $\mathrm{K}$, and then, it increases. The influences of the three factors in descending order are: the melt superheating temperature, the pouring temperature and the mold temperature. The optimized parameters for obtaining less delta ferrite are: the melt superheating temperature of $1893 \mathrm{~K}$, the pouring temperature of $1873 \mathrm{~K}$ and the mold temperature of $873 \mathrm{~K}$. It is very coincidental to find that the trend of the different parameters' effects on the fraction of delta ferrite is the same as that on the grain size, which means that the refinement can reduce the fraction of the delta ferrite in the X12 steel.

Based on the results above, considering the influence of the three parameters on the proportion of equiaxed grain 

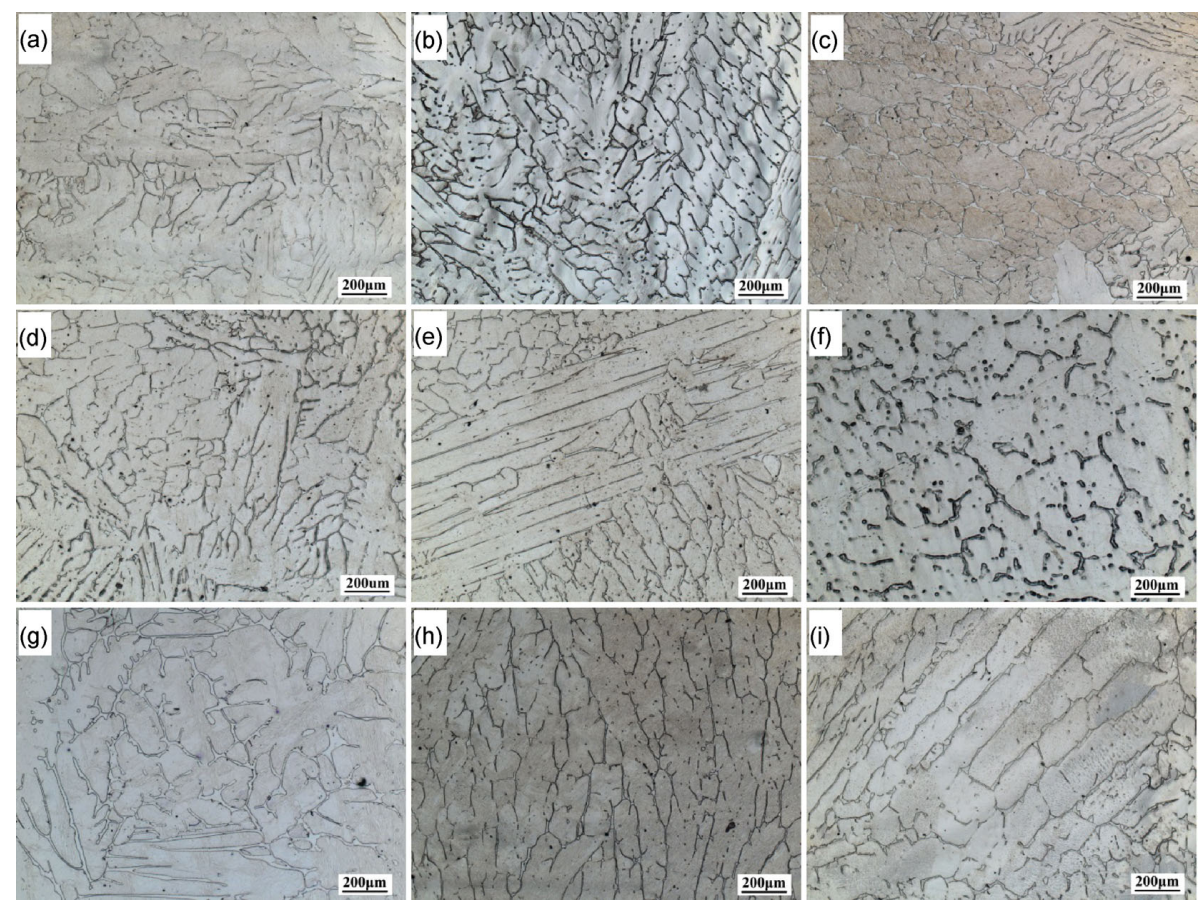

Fig. 4. The representative microstructure of $\mathrm{X} 12$ steel electrochemical polished in the orthogonal experiment. $T_{p}(\mathrm{~K}) / T_{m}$ (K)/Ts (K): (a)1 853/673/1 893, (b) $1853 / 873 / 1973$, (c) 1 853/1 073/2 053, (d) $1873 / 673 / 1$ 973, (e) $1873 / 873 / 2$ 053, (f) 1 873/1 073/1 893, (g)1 893,673/2 053, (h)1 893/873/1 893, (i)1 893/1 073/1 973. (Online version in color.)
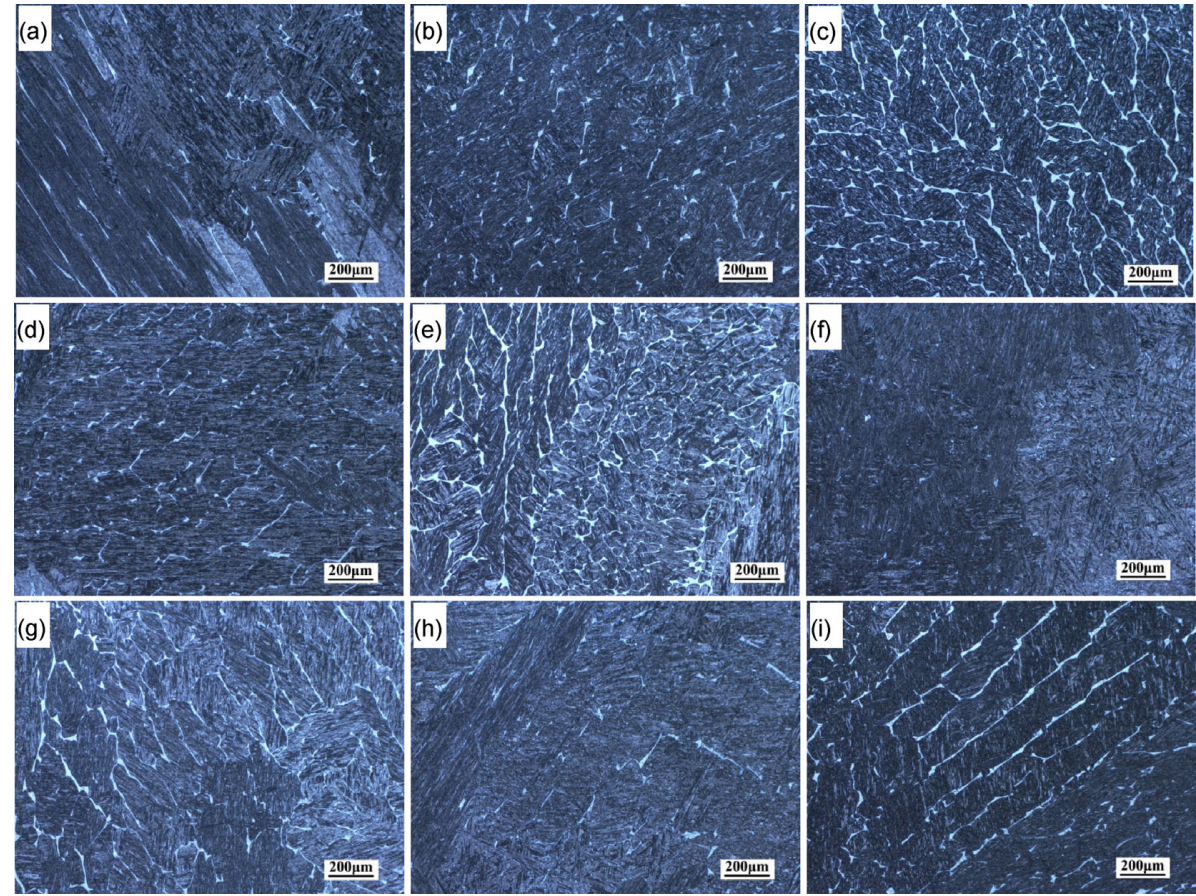

Fig. 5. The representative microstructure of X12 steel etched with Viella etchant reagent in the orthogonal experiment. $T_{p}$ (K)/T $T_{m}(\mathrm{~K}) / T_{s}$ (K): (a)1 853/673/1 893, (b)1 853/873/1 973, (c)1 853/1 073/2 053, (d)1 873/673/1 973, (e) $1873 / 873 /$ 2053, (f)1 873/1 073/1 893, (g)1 893,673/2 053, (h)1 893/873/1 893, (i) 1 893/1 073/1 973. (Online version in color.)

zone, the grain size and the fraction of delta ferrite, the optimized parameters for the sound ingot are $1893 \mathrm{~K}$ for the melt superheating temperature, $1073 \mathrm{~K}$ for the mold temperature and $1873 \mathrm{~K}$ for the pouring temperature.

\section{Discussion}

\subsection{Grain Refining Mechanisms}

The macrostructure of a solidified material is the result of the competition of columnar grains and equiaxed grains. ${ }^{14)}$ When the equiaxed crystals are small, they are swallowed by the approaching columnar front and transformed into columnar dendrites. Conversely, if the undercooled zone is relatively large and the density of the crystals is high, then the equiaxed crystals could have enough time to reach a sufficiently high volume fraction to block the columnar crystal growth. Spittle ${ }^{15)}$ has summarized the proposed mechanisms for termination of columnar growth. Among them, a criteri- 

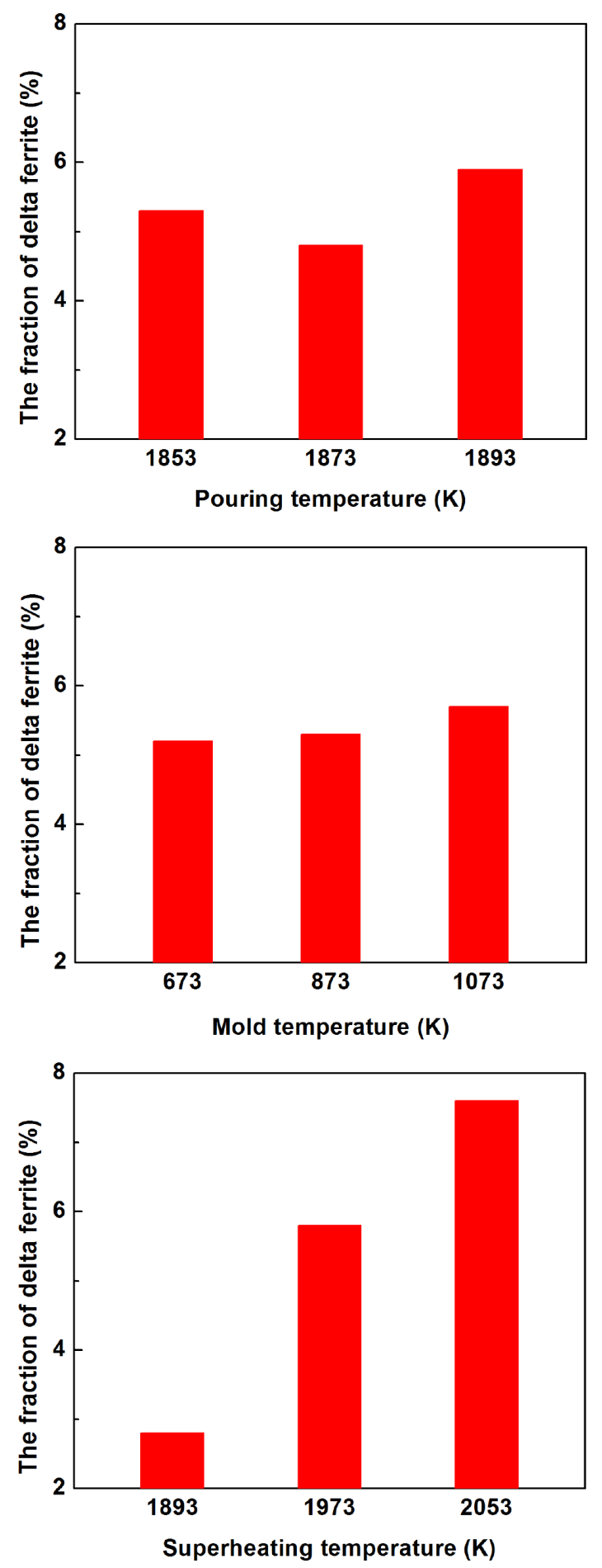

Fig. 6. Effects of parameters on the proportion of delta ferrite. (Online version in color.)

on proposed by Hunt ${ }^{16)}$ can be expressed as:

$$
G<0.617 N_{0}^{1 / 3}\left[1-\left(\Delta T_{N}\right)^{3} /\left(\Delta T_{C}\right)^{3}\right] \Delta T_{C}
$$

Where $G$ refers to the temperature gradient in the melt ahead of the solid-liquid interface; $N_{0}$ is the number of the heterogeneous sites per cubic meter; $\Delta T_{N}$ is the critical undercooling of heterogeneous nucleation; and $\Delta T_{C}$ is the undercooling in the melt ahead of the solid-liquid interface. From Eq. (2), it can be observed that increasing $N_{0}$ and $\Delta T_{C}$ and reducing $G$ can promote the transformation of coarse columnar grains to
Table 4. Effects of parameters on the fraction of delta ferrite.

\begin{tabular}{cccc}
\hline & $T_{p}(\mathrm{~K})$ & $T_{m}(\mathrm{~K})$ & $T_{s}(\mathrm{~K})$ \\
\hline$K_{1}$ & 5.3 & 5.2 & 2.8 \\
$K_{2}$ & 4.8 & 5.3 & 5.8 \\
$K_{3}$ & 5.9 & 5.7 & 7.6 \\
$R$ & 1.1 & 0.5 & 4.8 \\
\hline
\end{tabular}

fine equiaxed grains, which is favorable to the equiaxed fraction increment and grain refinement.

According to the research of Popel and Sidorov, ${ }^{17)}$ the initial metallic melt is inhomogeneous and can be considered to be a metastable non-equilibrium microemulsion or a microsuspension of disperse particles enriched in one of the components and surrounded by a molten matrix with a different composition. These particles $(\sim 10-100 \mathrm{~nm}$ in size) appear to have sharp but irregular interfaces. Their internal structure and local properties are more or less homogeneous. Furthermore, their dissolution in the surrounding melt is very slow, and it is favorable for the melt to nucleate on their interfaces. When heating a melt to a critical temperature (a temperature that is characteristic of its composition), the thermodynamic equilibrium between the particles and the melt matrix is destroyed and the system irreversibly transforms to a true solution state that is thermodynamically stable. ${ }^{18)}$ This transformation can be sharp but can also take place via several steps in the dispersion of the particles to smaller ones and after which the melt will conserve the microhomogeneous state during cooling down to liquid, and the influence of the melt will remain in the solid samples. Usually, it is impossible or rather difficult to overheat the high melting point alloys to a true solution using standard equipment and heterogeneous nucleation plays a leading role. In this work, $2053 \mathrm{~K}$ for the melt superheating temperature is not high enough to make the X12 steel melt change to a true solution state, and the number of particles that could act as heterogeneous nucleation sites $\left(N_{0}\right)$ becomes smaller with the increase in the superheating temperature. Therefore, the proportion of the equiaxed grain zone decreases but the grain size increases when the melt superheating temperature increases from $1893 \mathrm{~K}$ to $2053 \mathrm{~K}$, as indicated in Fig. 3(c).

Heating the mold at high temperature, the temperature gradient between the mold and the molten alloy is reduced, and the temperature gradient $(G)$ in the melt ahead of the solid-liquid interface is reduced also. Therefore, the proportion of the equiaxed grain zone increases, and the grain size decreases with the increase in the mold temperature.

It is suggested that the grains nucleate at the time of pouring in the thermally supercooled region beside the mold wall. ${ }^{15)}$ Poured at a low temperature, the molten alloy conducts less heat to the mold, and the nucleation starts immediately on the inner wall of the casting mold. Then, the heat flows from the molten alloy to the mold and the columnar zone forms. Poured at a high temperature, the molten alloy conducts much heat to the mold and the mold is heated to a high temperature. It could be assumed that there is a favorable pouring temperature (in our work, $1873 \mathrm{~K}$, for example) at which once the crystallization begins near the inner wall of the mold, the crystallites are melted by the high tem- 


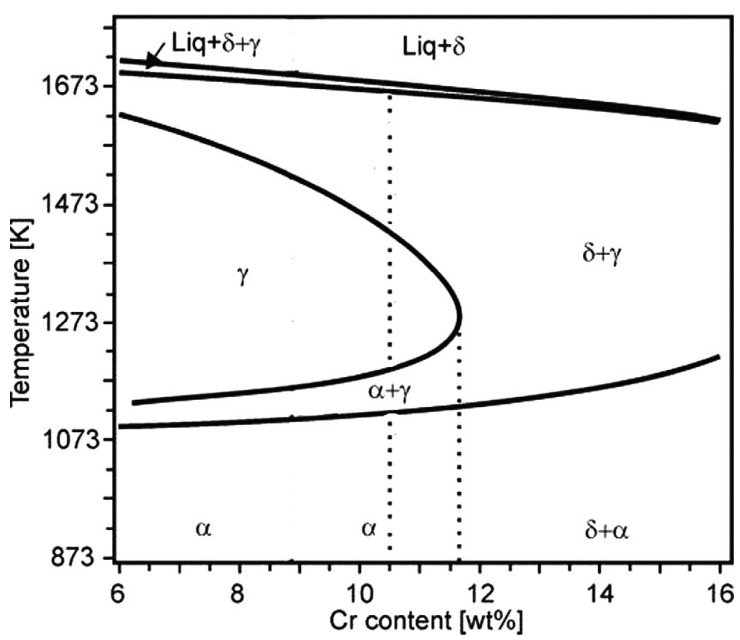

Fig. 7. An equilibrium phase diagram of $\mathrm{Fe}-\mathrm{Cr}$ alloys. ${ }^{19)}$

perature melt. Then, the solidification is delayed, and the temperature field in the melt distributes uniformly because the thermal conductivity of the steel is far greater than that of the sand mold. At the same time, the convection caused by pouring brings some unmelted crystallites into the bulk melt which accelerates the uniformity of the temperature field in the melt. Thus, the undercooled zone is enlarged, and the undercooling $\Delta T_{C}$ is amplified as the temperature decreases, which results in the grains of the ingot becoming homogenous and fine.

\subsection{Delta Ferrite Reduction Mechanisms}

Figure 7 is an equilibrium phase diagram of $\mathrm{Fe}-\mathrm{Cr}$ alloys calculated by Mayr et al. ${ }^{19)}$ It can be observed that a fully martensitic microstructure with no stable delta ferrite is formed at room temperature for the steel with the nominal $\mathrm{Cr}$ content (10.5 wt.\%) in the process of equilibrium solidification. In contrast, a martensitic matrix with a certain amount of stable delta ferrite is obtained when the Cr content in the steel increases beyond 11.4 wt.\%. During the non-equilibrium solidification process, the primary dendrites of the solid phase begin to push the solute atoms which are present in the highest concentration in the steel, mainly chromium in X12 steel, toward the interdendritic regions containing the liquid phase. ${ }^{13)}$ The liquid becomes increasingly rich in chromium as the temperature decreases, thus promoting delta ferrite phase solidification in these regions. It is obvious that the coarser the grain is, the more solidification time it takes, which means that more solute atoms are pushed to the interdendritic regions. Therefore, a larger fraction of delta ferrite is obtained at room temperature.

\section{Conclusions}

In this paper, $\mathrm{X} 12 \mathrm{CrMoWVNbN10-1-1} \mathrm{ingots} \mathrm{were} \mathrm{pre-}$ pared in a group of orthogonal experiments. The effects of the melt pouring temperature, mold temperature and melt superheating temperature on the microstructure of the X12 steel ingots were investigated, and the conclusions were summarized as follows:

(1) The number of particles that could act as heterogeneous nucleation sites $\left(N_{0}\right)$ increased when the melt superheating temperature decreased from $2053 \mathrm{~K}$ to $1893 \mathrm{~K}$; therefore, both the grain size and the proportion of delta ferrite decreased while the proportion of the equiaxed grain zone increased.

(2) The proportion of the equiaxed grain zone expanded when the mold temperature increased from $873 \mathrm{~K}$ to 1073 $\mathrm{K}$, which resulted from a reduction in the temperature gradient $(G)$ in the melt ahead of the solid-liquid interface, while the grain size and the proportion of ferrite changed imperceptibly.

(3) At a favorable pouring temperature of $1873 \mathrm{~K}$, the ingot became more uniform and fine and exhibited less delta ferrite. This result was observed primarily because of the enlargement of the undercooled zone and the augmentation of the undercooling $\Delta T_{C}$.

(4) The optimized parameters for a sound ingot were $1893 \mathrm{~K}$ for the melt superheating temperature, $1073 \mathrm{~K}$ for the mold temperature and $1873 \mathrm{~K}$ for the pouring temperature. Under that condition, the ingot was composed of fine equiaxed grain with radius of $1.1 \mathrm{~mm}$, and the fraction of delta ferrite was less than $2 \%$.

\section{Acknowledgements}

The authors are grateful for the financial support of National Basic Research Program of China (No. 2011CB 12900) and National Natural Science Foundation of China (No.51174134). The authors would also like to thank Dr. Xiaoying Shi for the helpful discussion.

\section{REFERENCES}

1) Y. Tanaka and I. Sato: J. Nucl. Mater., 417 (2011), 854.

2) K. Nakajima, H. Hasegawa, S. Khumkoa and S. Mizoguchi: Metall. Mater. Trans. B, 34 (2003), 539.

3) W. J. Poole, A. Mitchell and F. Weinberg: High Temp. Mater. Proc., 16 (1997), 173.

4) H. T. Zhang, H. Nagaumi and J. Z. Cui: Mater. Sci. Eng. A, 448 (2007), 177.

5) X. P. Ma, Y. J. Li and Y. S. Yang: J. Mater. Res., 24 (2009), 2670.

6) Q. M. Liu, Q. J. Zhai, F. P. Qi and Y. Zhang: Mater. Lett., 61 (2007), 2422 .

7) W. Michael and B. Howard: Proc. of the Superalloys, AIME, PA, (1984), 3.

8) A. Kumar, K. Laha, T. Jayakumar, K. B. S. Rao and B. Raj: Metall. Mater. Trans. A, 33 (2002), 1617.

9) K. Sawada, H. Kushima and K. Kimura: ISIJ Int., 46 (2006), 769.

10) B. Z. Xia: The Orthogonal Experiments Design Method, Jilin People's Publishing House, Jilin, (1985), 2 (in Chinese).

11) Z. Y. Pei, J. T. Li, Y. W. Tian and M. H. Zhao: Mater. Rev., 20 (2006), 83 (in Chinese).

12) Z. Q. Cui and B. X. Liu: Physical Metallurgy and Principle of Heattreatment, Harbin Institute of Technology Press, Harbin, (2007), 103 (in Chinese).

13) M. Martins and P. E. C. de Cerqueira: Int. J. Cast Met. Res., 25 (2012), 121 .

14) C. Y. Wang and C. Beckermann: Metall. Mater. Trans. A, 25A (1994), 1081.

15) J. A. Spittle: Int. Mater. Rev., 51 (2006), 247.

16) J. D. Hunt: Mater. Sci. Eng. A, 65 (1984), 75.

17) P. E. Popel and V. E. Sidorov: Mater. Sci. Eng. A, 226-228 (1997), 237.

18) V. E. Sidorov, M. Calvo-Dahlborg, U. Dahlborg, P. S. Popel and S. Chernoborodova: J. Mater. Sci., 35 (2005), 2255.

19) P. Mayr, T. A. Palmer, J. W. Elmer, E. D. Specht and S. M. Allen: Metall. Mater. Trans. A, 41 (2010), 2462. 